\title{
CARACTERIZAÇÃO FÍSICA E QUÍMICA, FENÓLICOS TOTAIS E ATIVIDADE ANTIOXIDANTE DA POLPA E RESÍDUO DE GABIROBA ${ }^{1}$
}

\author{
ALINE MEDEIROS ALVES², MARESSA STEPHANIE OVIDIO ALVES 3 , \\ THAÍS DE OLIVEIRA FERNANDES ${ }^{4}$, RONALDO VELOSO NAVES ${ }^{5}$, \\ MARIA MARGARETH VELOSO NAVES 6
}

RESUMO - O Cerrado brasileiro possui uma riqueza de espécies frutíferas que ainda não foram suficientemente estudadas em relação às suas características físicas, químicas e funcionais. O presente estudo teve o objetivo de mensurar as características físicas de frutos de gabirobeira e analisar a composição centesimal e de minerais, o teor de compostos fenólicos totais e a atividade antioxidante da polpa e do resíduo de gabiroba. As características físicas de maior variabilidade foram massa da polpa e do fruto, destacando-se o elevado rendimento de polpa (46,24\%). A polpa e o resíduo de gabiroba contêm altos teores de umidade e fibra alimentar e quantidades consideráveis de ferro. O resíduo de gabiroba apresentou maior conteúdo de compostos fenólicos $(1.787,65 \mathrm{mg}$ AGE. $\left.100 \mathrm{~g}^{-1}\right)$ e atividade antioxidante $\left(197,13 \mu \mathrm{mol} \mathrm{TE} \cdot \mathrm{g}^{-1}\right)$ em relação à polpa. Contudo, os valores constatados na polpa de gabiroba $\left(1.222,59 \mathrm{mg}\right.$ AGE. $100 \mathrm{~g}^{-1}$ e $107,96 \mu \mathrm{mol} \mathrm{TE} . \mathrm{g}^{-1}$, respectivamente) são superiores aos de muitos frutos consumidos tradicionalmente. $\mathrm{O}$ teor de fenólicos totais apresentou forte correlação $(\mathrm{r}=0,9723)$ com a atividade antioxidante. Os resultados indicam perspectivas promissoras para o aproveitamento integral do fruto da gabirobeira, visto seu conteúdo apreciável de nutrientes e de compostos fenólicos, e sua elevada atividade antioxidante.

Termos para indexação: Campomanesia adamantium (Cambess.) O. Berg, composição centesimal, minerais, compostos bioativos, capacidade antioxidante.

\section{PHYSICAL AND CHEMICAL CHARACTERIZATION, TOTAL PHENOLICS AND ANTIOXIDANT ACTIVITY OF THE GABIROBA PULP AND RESIDUE}

\begin{abstract}
The Brazilian Cerrado has a wide range of fruit species that have not been sufficiently studied regarding their physical, chemical and functional characteristics. This study aimed to measure the physical characteristics of gabiroba tree's fruits and analyze the proximate and mineral compositions, total phenolic content and antioxidant activity of the gabiroba pulp and residue. The physical characteristics of greatest variability were pulp and fruit mass, especially the high pulp yield (46.24\%). Gabiroba pulp and residue have high moisture and dietary fiber contents and considerable iron amounts. The gabiroba residue showed higher content of phenolic compounds (1787.65 mg AGE. $\left.100 \mathrm{~g}^{-1}\right)$ and antioxidant activity $\left(197.13 \mu \mathrm{mol} \mathrm{TE. \textrm {g } ^ { - 1 } )}\right.$ than the pulp. However, the values observed in the gabiroba pulp (1222.59 mg AGE. $100 \mathrm{~g}^{-1}$ and $107.96 \mu \mathrm{mol} \mathrm{TE.g^{-1 }}$, respectively) are higher than those of many fruits traditionally consumed. The total phenolic content showed a strong correlation $(r=0.9723)$ with antioxidant activity. The results indicate promising prospects for the use of the whole fruit of gabiroba tree due to its appreciable content of nutrients and phenolic compounds, and its antioxidant activity. Index terms: Campomanesia adamantium (Cambess.) O. Berg, proximate composition, minerals, bioactive compounds, antioxidant capacity.
\end{abstract}

\footnotetext{
1(Trabalho 081-13). Recebido em: 28-02-2013. Aceito para publicação em: 06-09-2013.

${ }^{2}$ Mestranda em Ciência e Tecnologia de Alimentos, Escola de Agronomia e Engenharia de Alimentos, Universidade Federal de Goiás (UFG), Goiânia-GO, Brasil .E-mail: amedeiros87@gmail.com

${ }^{3}$ Graduanda em Nutrição, Faculdade de Nutrição, UFG, Goiânia-GO, Brasil. E-mail: maressa.av@gmail.com

${ }^{4}$ Mestranda em Nutrição e Saúde, Faculdade de Nutrição, UFG. E-mail: thaisfernandes.nut@gmail.com

${ }^{5}$ Doutor em Produção Vegetal, Docente da Escola de Agronomia e Engenharia de Alimentos, UFG. E-mail: ronaldo@agro.ufg.br

${ }^{6}$ Doutora em Ciência dos Alimentos, Docente da Faculdade de Nutrição, UFG. E-mail: mmvnaves@gmail.com
} 


\section{INTRODUÇÃO}

A gabirobeira (Campomanesia sp.) é uma planta de ampla distribuição no Cerrado, podendo ser encontrada em vários estados brasileiros, com maior concentração no Estado de Goiás. Seu florescimento ocorre de agosto a novembro, e a frutificação, de setembro a dezembro, podendo estender-se até fevereiro. $\mathrm{O}$ fruto da gabirobeira, a gabiroba, também conhecida como guabiroba, guabiroba-do-campo e guavira, caracteriza-se por ser um fruto arredondado, de coloração amarelo-esverdeada, constituído por uma casca fina e uma polpa esbranquiçada, envolvendo diversas sementes (VALLILO et al., 2006).

Quanto à composição nutricional, a gabiroba apresenta elevados teores de umidade e fibra alimentar, resultando em baixa densidade energética (cerca

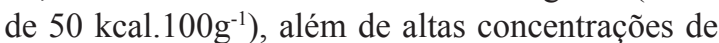
potássio, fósforo, magnésio e ferro (SILVA et al., 2008; VALLILO et al., 2006). Ainda, contém quantidades apreciáveis de compostos bioativos, como o ácido ascórbico e os compostos fenólicos (PEREIRA et al., 2012; ROCHA et al., 2011), sugerindo boa atividade antioxidante.

Estudos indicam que o consumo diário de frutas pode contribuir para promoção e prevenção de diversas doenças, em decorrência do crescente reconhecimento do valor nutricional e terapêutico desses alimentos (JOSHIPURA et al., 2009; SOERJOMATARAM et al., 2010). Por outro lado, a busca por substâncias naturais, biologicamente ativas e de baixo custo, tem elevado o número de pesquisas com os resíduos do processamento das frutas, como casca e semente, que são considerados fontes potenciais de nutrientes. Há relatos de que a concentração de nutrientes nos resíduos das frutas é maior do que na polpa (IGNAT et al., 2011). Dentre os compostos presentes nas frutas e em seus resíduos, aqueles com ação antioxidante têm despertado o interesse da comunidade científica, como alguns minerais e vitaminas e os compostos fenólicos.

A caracterização física e química de frutas nativas do Cerrado e seus resíduos e a quantificação de compostos bioativos também contribuem para agregar valor e potencializar o uso comercial e industrial destas frutas, bem como para a conservação deste bioma. Sendo assim, este trabalho teve o objetivo de analisar as características físicas e químicas, o conteúdo de fenólicos totais e a atividade antioxidante da polpa e do resíduo de gabiroba.

\section{MATERIAL E MÉTODOS}

Aquisição dos frutos e processamento das amostras

Os frutos foram coletados na região central do Estado de Goiás $\left(16^{\circ} 19^{\prime} 37^{\prime \prime}\right.$ a $16^{\circ} 58^{\prime} 5^{\prime \prime} \mathrm{S} ; 48^{\circ}$ $57^{\prime} 10^{\prime \prime}$ a $49^{\circ} 13^{\prime} 54^{\prime \prime} \mathrm{O}$ ), em áreas com vegetação típica dos Cerrados, no mês de novembro de 2011. O material estudado foi identificado e registrado no Herbário da Universidade Federal de Goiás, sob o número 47620.

Após a coleta, os frutos foram selecionados de acordo com a cor, maturação, textura e ausência de injúrias, e lavados em água corrente. Em seguida, os frutos foram despolpados em despolpadeira industrial (0.25 DF, Bonina) para separar a polpa da casca e da semente. Posteriormente, as frações polpa e resíduo (casca e semente) foram homogeneizadas e liofilizadas em liofilizador (L108, Liotop). Após a liofilização, as amostras foram embaladas a vácuo e armazenadas sob refrigeração, com luminosidade controlada, até o momento das análises químicas.

\section{Caracterização física}

Após a coleta, 100 frutos in natura foram selecionados aleatoriamente para as análises físicas. Com o auxílio de balança semianalítica (BG8000, Gehaka) e paquímetro digital (500-144B, Mitutoyo), as seguintes variáveis físicas foram determinadas: massa do fruto, da polpa e do resíduo, e diâmetros longitudinal e transversal. O rendimento de polpa foi determinado pela relação entre a massa da polpa e a massa do fruto.

\section{Composição centesimal e de minerais}

A composição centesimal das frações da gabiroba foi determinada por meio das análises de: umidade; nitrogênio total e sua conversão em proteína bruta, utilizando-se do fator 6,25; cinzas; fibra alimentar total - solúvel e insolúvel (AOAC, 2002); e lipídios totais (BLIGH; DYER, 1959). O teor de carboidratos totais foi estimado por diferença, subtraindo-se de cem os valores obtidos para umidade, proteínas, lipídios, cinzas e fibra alimentar total. A composição centesimal permitiu estimar o valor energético das amostras, considerandose os fatores de conversão de Atwater, de 4; 4 e $9 \mathrm{kcal.g}{ }^{-1}$ para proteína, carboidratos e lipídios, respectivamente.

As cinzas das amostras foram solubilizadas em ácido clorídrico concentrado. Os teores dos 
minerais, cálcio, ferro e zinco foram quantificados por meio de espectrometria de absorção atômica, e os conteúdos de sódio e potássio, por emissão atômica, utilizando-se do espectrômetro Analyst-200 (Perkin Elmer, Waltham, EUA) (AOAC, 2002).

\section{Preparo dos extratos}

A extração foi realizada homogeneizando-se $0,5 \mathrm{~g}$ de amostra liofilizada em $25 \mathrm{~mL}$ de metanol/ água $(70: 30 \mathrm{v} / \mathrm{v})$, em agitador magnético RO 10 (IKA, Staufen, Alemanha), por 120 minutos a $4^{\circ} \mathrm{C}$. O extrato obtido foi posteriormente filtrado em papel de filtro e acondicionado em frasco de vidro âmbar até o momento da análise (ABE et al., 2010). Este extrato foi utilizado para determinar o conteúdo de compostos fenólicos totais e a atividade antioxidante.

\section{totais}

\section{Determinação de compostos fenólicos}

A determinação de compostos fenólicos totais foi conduzida conforme procedimento descrito por Singleton e Rossi (1965), com modificações. Uma alíquota de $0,25 \mathrm{~mL}$ dos extratos foi misturada com $2,5 \mathrm{~mL}$ de água e $0,25 \mathrm{~mL}$ do reagente de FolinCiocalteu. Após 5 minutos à temperatura ambiente, $0,25 \mathrm{~mL}$ de solução de carbonato de sódio $(10 \%)$ foi adicionada, e a mistura, mantida à temperatura ambiente, com luminosidade controlada, por 60 minutos. A absorbância a $725 \mathrm{~nm}$ foi determinada em espectrofotômetro UV/Visível V-630 (Jasco, Tóquio, Japão), e o conteúdo de fenólicos totais foi calculado por meio de curva-padrão de ácido gálico (16 a $100 \mathrm{mg} / \mathrm{L})$. Os resultados foram expressos em $\mathrm{mg}$ de ácido gálico equivalente por $100 \mathrm{~g}$ de peso fresco (mg AGE.100g ${ }^{-1}$ p.f.).

\section{Determinação da atividade antioxidante Método ABTS (2,2'-azino-bis (3-etilbenzo- tiazolina) 6-ácido sulfônico)}

A atividade antioxidante equivalente ao Trolox foi determinada de acordo com procedimento proposto por Re et al. (1999), com algumas modificações. O radical ABTS foi preparado a partir da reação de $7 \mathrm{mM}$ de solução aquosa de ABTS com $140 \mathrm{mM}$ de persulfato de potássio, deixando-se a mistura à temperatura ambiente por 16 horas, na ausência de luz. Em seguida, a solução de ABTS foi diluída com etanol para obter uma absorbância de $0,70 \pm 0,05$ a $734 \mathrm{~nm}$. Alíquotas de $30 \mu \mathrm{L}$ das amostras foram adicionadas a $3 \mathrm{~mL}$ da solução diluída de ABTS, e as absorbâncias da mistura foram registradas ao final de seis minutos. A atividade antioxidante foi calculada, utilizando-se da curvapadrão de Trolox (100 a $2000 \mu \mathrm{M})$ e suas respectivas porcentagens de inibição, e os resultados do ensaio foram expressos em $\mu \mathrm{mol}$ de Trolox equivalente por grama de peso fresco ( $\mu \mathrm{mol} \mathrm{TE} . \mathrm{g}^{-1}$ p.f.).

\section{Análise estatística}

As análises foram realizadas em triplicata, e os resultados foram submetidos ao teste $t$ de Student para comparação de médias $(\mathrm{p}<0,05)$. A correlação entre o conteúdo de fenólicos totais e a atividade antioxidante foi determinada por meio do coeficiente de correlação de Pearson. Os cálculos estatísticos foram efetuados pelo programa Statistica 7.0.

\section{RESULTADOS E DISCUSSÃO}

Os frutos de $C$. adamantium apresentaram massa média e diâmetros longitudinal e transversal (Tabela 1) superiores aos valores constatados por Oliveira et al. (2011), para frutos de $C$. adamantium e $C$. pubescens, procedentes do município de Uberlândia, Minas Gerais. O rendimento de polpa foi elevado, resultado que pode contribuir para a seleção de frutos com maior rendimento, potencializando o uso comercial e industrial deste fruto. Dentre as características estudadas, observou-se maior variabilidade para as seguintes variáveis: massa dos frutos $(\mathrm{CV}=27,90 \%) \mathrm{e}$ massa de polpa $(\mathrm{CV}=36,18 \%)$. Essas variações podem estar relacionadas à fisiologia de cada espécie estudada, às condições climáticas da região de origem e ao tipo de solo (OLIVEIRA et al., 2011).

A composição centesimal revelou que a polpa e o resíduo da gabiroba apresentam teores elevados de umidade e baixos valores energéticos (Tabela 2). Resultados compatíveis foram relatados por Silva et al. (2008), para polpa de gabiroba, e por Vallilo et al. (2006), para frutos inteiros de C. adamantium provenientes da região oeste do Estado de São Paulo. Constatou-se que os teores de lipídios, proteínas e cinzas no resíduo de gabiroba foram superiores aos valores encontrados na polpa (Tabela 2), e próximos dos relatados para casca de manga (MARQUES et al., 2010). Ressalta-se que não foram encontrados na literatura dados sobre a composição química das porções não comestíveis (casca e semente) desse fruto.

O conteúdo de fibra alimentar total (Tabela 2) das frações da gabiroba variou de 7 a 24 g. $100 \mathrm{~g}^{-1}$, o que perfaz $23 \%$ a $80 \%$ da recomendação diária de fibra alimentar para um indivíduo adulto saudável (30 g) (IOM, 2006), constituindo-se em um alimento com alto teor de fibra, que é capaz de reduzir os níveis séricos de triglicerídeos e glicose (KACZMARCZYK 
et al., 2012). O conteúdo de fibra alimentar no resíduo de gabiroba foi cerca de três vezes maior que o teor observado na polpa (Tabela 2), e em frutos inteiros de $C$. adamantium $\left(9,0 \mathrm{~g} .100 \mathrm{~g}^{-1}\right)$ (VALLILO et al., 2006). O teor de fibra alimentar do resíduo de gabiroba também foi superior ao relatado para casca de manga (11,02 g.100g-1) (MARQUES et al., 2010).

Vale acrescentar que as porções não comestíveis de frutas e hortaliças são tradicionalmente descartadas, gerando grandes quantidades de lixo orgânico. Como observado em nosso estudo, outros trabalhos também mostram que esses resíduos possuem mais nutrientes do que as polpas, podendo ser fontes importantes de nutrientes e compostos bioativos (IGNAT et al., 2011). Portanto, recomenda-se a inserção dessas porções na dieta, desde que seja avaliada a presença de fatores antinutricionais e de compostos tóxicos, como metais pesados e defensivos agrícolas.

Em relação aos minerais (Tabela 3), as duas frações da gabiroba apresentaram maiores concentrações de cálcio e potássio, em relação aos teores de ferro, sódio e zinco. Considerando os elementos inorgânicos analisados, Vallilo et al. (2006) também observaram maiores quantidades de cálcio e potássio em frutos inteiros de C. adamantium. Entretanto, o ferro foi o mineral que apresentou maior contribuição para Ingestão Dietética Recomendada (Recommended Dietary Allowance - RDA) em relação a uma porção de $100 \mathrm{~g}$ de fruto. Destaca-se que esse mineral é essencial para a maioria dos organismos vivos, pois atua como cofator de enzimas em inúmeros processos, como homeostase celular, síntese de proteínas e transporte de oxigênio (SHEFTEL et al., 2012). O teor desse mineral encontrado na polpa de gabiroba foi próximo do observado para polpa de maracujá-do-cerrado $(1,06$ $\left.\mathrm{mg} .100 \mathrm{~g}^{-1}\right)$, e superior ao relatado para polpa de murici (0,17 mg.100 $\left.\mathrm{g}^{-1}\right)$ (SOUZA et al., 2012). Os teores de minerais do resíduo de gabiroba foram maiores do que os da polpa, exceto para o conteúdo de ferro, que não apresentou diferença significativa entre as duas frações (Tabela 3).

O teor de fenólicos totais da polpa de gabiroba foi de 1.222,59 mg AGE.100g $\mathrm{g}^{-1}$ (Figura 1), sendo superior aos valores relatados para polpa de frutos de Campomanesia sp. procedentes dos Estados de Goiás e Distrito Federal (259 a 285 mg AGE. 100 $^{-1}$ ) (ROCHA et al., 2011). O conteúdo de fenólicos totais constatados em polpas de frutas tropicais, como a acerola $(1.063 \mathrm{mg}$ AGE. $\left.100 \mathrm{~g}^{-1}\right)$ e o camu-camu (1.176 mg AGE.100g $\mathrm{g}^{-1}$ ) (RUFINO et al., 2010), e em polpas de frutos procedentes do Cerrado, como murici (334,37 mg AGE. $\left.100 \mathrm{~g}^{-1}\right) \mathrm{e}$ marolo (739,37 mg AGE.100g-1 ${ }^{-1}$ (SOUZA et al., 2012), são inferiores ao valor observado para polpa de gabiroba no presente estudo. Sabe-se que a concentração de compostos fenólicos de um alimento pode variar conforme as condições geográficas e ambientais da região de origem e os fatores fisiológicos e genéticos da planta (MARTINS et al., 2011). Esses fatores podem explicar as diferenças observadas entre o teor de fenólicos da polpa de gabiroba de nosso estudo com os descritos na literatura. O conteúdo de fenólicos totais do resíduo de gabiroba foi de 1.787,65 mg AGE.100 $\mathrm{g}^{-1}$ (Figura 1), resultado superior aos valores observados para casca de jatobá (Hymenaea courbaril) (1.712 mg AGE. $100 \mathrm{~g}^{-1}$ ) e semente de araçá-boi (Eugenia estipitata) (1.624 mg AGE.100g ${ }^{-1}$ ) (CONTRERAS-CALDERÓN et al., 2011).

Os compostos fenólicos são substâncias essenciais para o crescimento e a reprodução dos vegetais. Além disso, são responsáveis pela pigmentação e pela proteção contra raios ultravioleta, microrganismos e insetos. As plantas, quando submetidas a situações de estresse, como limitações nutricionais ou ataques de patógenos, ativam as rotas de síntese dos metabólitos secundários, resultando na produção dos compostos fenólicos (IGNAT et al., 2011). As condições ambientais do bioma Cerrado, como solos pobres em nutrientes (CARVALHO et al., 2009), favorecem essas situações de estresse, o que justifica um acúmulo maior desses compostos nas partes externas dos frutos de gabirobeira.

No organismo humano, os compostos fenólicos podem atuar na eliminação de radicais livres, proteção de antioxidantes dietéticos (vitamina $\mathrm{E}$ e C) e complexação de íons metálicos (MARTINS et al., 2011), promovendo benefícios adicionais à saúde. Por causa dessas propriedades e do conteúdo elevado de compostos fenólicos no resíduo e na polpa de gabiroba, sugere-se a aplicação deste fruto nos setores alimentício e farmacêutico.

A atividade antioxidante das frações da gabiroba foi determinada pelo método ABTS, que se fundamenta na redução do radical monocátion ABTS pelas substâncias antioxidantes. O valor observado de atividade antioxidante da polpa de gabiroba $(107,96$ $\mu$ mol TE. $\mathrm{g}^{-1}$ ) (Figura 1) foi próximo dos valores relatados para atividade antioxidante, pelo método ABTS, de polpa de frutos como puçá-preto $\left(125 \mu \mathrm{mol} \mathrm{TE} . \mathrm{g}^{-1}\right)$ e murici $\left(131,58 \mu \mathrm{mol} \mathrm{TE} . \mathrm{g}^{-1}\right)$ (RUFINO et al., 2010; SOUZA et al., 2012). A atividade antioxidante do resíduo de gabiroba $\left(197,13 \mu \mathrm{mol} \mathrm{TE}^{-1} \mathrm{~g}^{-1}\right)$ foi superior à da polpa (Figura 1) e à relatada para casca de cupuaçu (Theobroma grandiflorum) $\left(65,3 \mu \mathrm{mol} \mathrm{TE.g^{-1 } )}\right.$ (CONTRERAS-CALDERÓN et al., 2011).

Em estudo realizado com frutos pertencentes à família Myrtaceae (PEREIRA et al., 2012), como goiaba-amarela, gabiroba e uvaia, a gabiroba (fruto inteiro) apresentou maior conteúdo de fenólicos totais 
e atividade antioxidante, pelo método ABTS. Assim como em nosso estudo, Pereira et al. (2012) observaram que esses resultados foram superiores aos de muitos frutos tradicionalmente consumidos. Não foram encontrados na literatura dados de atividade antioxidante da gabiroba expressos em unidade de concentração semelhante à do nosso estudo.

O conteúdo de fenólicos totais correlacionou- -se de forma positiva com a atividade antioxidante $(r=0,9723 ; p \leq 0,05)$, indicando a importância dos compostos fenólicos como determinantes da atividade antioxidante da gabiroba. Nesse sentido, diversos autores relatam elevada correlação entre o conteúdo de fenólicos totais e a atividade antioxidante (avaliada pelo método ABTS) de frutas (ALMEIDA et al., 2011; RUFINO et al., 2010; SOUZA et al., 2012).

TABELA 1 - Características físicas de frutos de gabirobeira (Camponesia adamantium) nativa do Cerrado.

\begin{tabular}{lccc}
\hline Variáveis & Mínimo & Máximo & Média $\pm \mathrm{DP}^{1}$ \\
\hline Diâmetro longitudinal $(\mathrm{mm})$ & 18,42 & 28,41 & $22,97 \pm 2,12$ \\
Diâmetro transversal $(\mathrm{mm})$ & 19,56 & 30,48 & $24,20 \pm 2,45$ \\
Massa (g) & & & \\
Fruto & 3,06 & 12,94 & $6,99 \pm 1,95$ \\
Polpa & 1,23 & 6,63 & $3,28 \pm 1,19$ \\
Resíduo (casca e semente) & 1,63 & 6,72 & $3,71 \pm 0,95$ \\
Rendimento de polpa (\%) & 20,03 & 66,03 & $46,24 \pm 6,78$ \\
\hline
\end{tabular}

${ }^{1}$ Média de 100 frutos; DP: desvio-padrão.

TABELA 2 - Composição centesimal e valor energético total da polpa e resíduo de gabiroba (Camponesia adamantium).

\begin{tabular}{|c|c|c|}
\hline \multirow{2}{*}{$\begin{array}{l}\text { Composição centesimal } \\
\text { (g.100g-1de peso fresco) }\end{array}$} & \multicolumn{2}{|c|}{ Gabiroba } \\
\hline & polpa & resíduo (casca e semente) \\
\hline Umidade & $80,87 \pm 0,04 a$ & $63,70 \pm 0,03 b$ \\
\hline Proteína & $1,06 \pm 0,00 \mathrm{~b}$ & $3,17 \pm 0,04 \mathrm{a}$ \\
\hline Lipídios & $0,55 \pm 0,03 b$ & $5,33 \pm 0,02 \mathrm{a}$ \\
\hline Carboidratos totais & $10,00 \pm 0,06 \mathrm{a}$ & $3,00 \pm 0,15 b$ \\
\hline Fibra alimentar total & $7,10 \pm 0,03 b$ & $24,05 \pm 0,15 \mathrm{a}$ \\
\hline Fibra solúvel & $1,49 \pm 0,03 b$ & $3,48 \pm 0,08 \mathrm{a}$ \\
\hline Fibra insolúvel & $5,60 \pm 0,02 b$ & $20,58 \pm 0,09 a$ \\
\hline Cinzas & $0,43 \pm 0,02 b$ & $0,74 \pm 0,01 \mathrm{a}$ \\
\hline VET (kcal.100g-1) & 49,19 & 72,65 \\
\hline
\end{tabular}

Valores constituem média \pm desvio-padrão. Médias com letras iguais na mesma linha não apresentam diferenças significativas pelo teste $t$ de Student $(\mathrm{p}<0,05)$. 
TABELA 3 - Composição em minerais da polpa e resíduo de gabiroba (C. adamantium).

\begin{tabular}{lcc}
\hline \multirow{2}{*}{ Minerais (mg.100g-1 de peso fresco) } & \multicolumn{2}{c}{ Gabiroba } \\
\cline { 2 - 3 } Cálcio & polpa & resíduo (casca e semente) \\
RDA $(\%)^{2}$ & $8,77 \pm 0,04^{\mathrm{b}}$ & $31,53 \pm 0,78^{\mathrm{a}}$ \\
Ferro & 0,88 & 3,15 \\
RDA (\%) & $1,07 \pm 0,03^{\mathrm{a}}$ & $1,08 \pm 0,02^{\mathrm{a}}$ \\
Sódio & 13,38 & 13,50 \\
RDA (\%) & $0,83 \pm 0,13^{\mathrm{b}}$ & $1,52 \pm 0,13^{\mathrm{a}}$ \\
Potássio & 0,05 & 0,10 \\
RDA (\%) & $121,71 \pm 2,52^{\mathrm{b}}$ & $257,47 \pm 6,10^{\mathrm{a}}$ \\
Zinco & 2,60 & 5,48 \\
RDA (\%) & $0,16 \pm 0,01^{\mathrm{b}}$ & $0,67 \pm 0,00^{\mathrm{a}}$ \\
\hline
\end{tabular}

${ }^{1}$ Valores constituem média \pm desvio-padrão. Médias com letras iguais na mesma linha não apresentam diferenças significativas, pelo teste $\mathrm{t}$ de Student $(\mathrm{p}<0,05) .{ }^{2}$ Recommended Dietary Allowance (RDA) para adultos saudáveis (31 a 50 anos): cálcio= $1.000 \mathrm{mg} / \mathrm{dia}$; ferro $=8 \mathrm{mg} /$ dia; sódio $=1.500 \mathrm{mg} /$ dia; potássio $=4.700 \mathrm{mg} / \mathrm{dia} ;$ zinco $=11 \mathrm{mg} / \mathrm{dia}(\mathrm{IOM}, 2006)$.
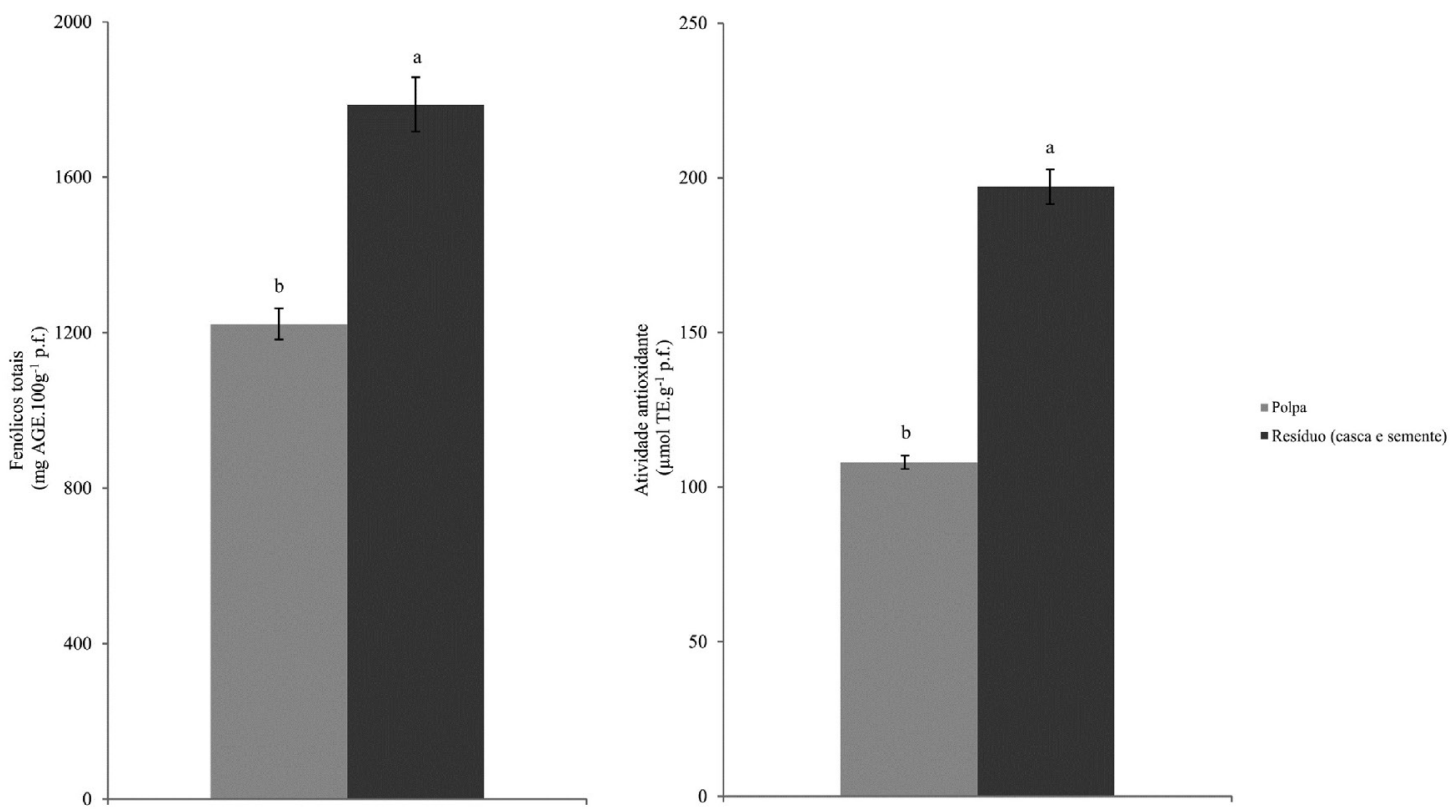

FIGURA 1 - Conteúdo de compostos fenólicos totais e atividade antioxidante, pelo método ABTS, da polpa e resíduo de gabiroba (C. adamantium).

${ }^{\mathrm{a}, \mathrm{b}}$ Letras diferentes representam diferenças significativas entre médias, pelo teste $\mathrm{t}$ de Student $(\mathrm{p}<0,05)$; AGE: ácido gálico equivalente; TE: trolox equivalente; p.f.: peso fresco. 


\section{CONCLUSÃO}

Considerando as características físicas, nutricionais e funcionais da gabiroba, recomenda-se o consumo deste fruto e a inserção de seus resíduos na alimentação, de forma adequada, para contribuir com a ingestão diária de antioxidantes e proteção do organismo contra os danos oxidativos. Entretanto, estudos adicionais são necessários para promover o cultivo de gabiroba em escala comercial.

\section{AGRADECIMENTOS}

Ao Conselho Nacional de Desenvolvimento Científico e Tecnológico (CNPq), pelo apoio financeiro.

\section{REFERÊNCIAS}

ABE, L. T.; LAJOLO, F. M.; GENOVESE, M. I. Comparison of phenol content and antioxidant capacity of nuts. Ciência e Tecnologia de Alimentos, Campinas, v. 30, Supl. 1, p. 254-259, 2010.

ALMEIDA, M. M. B.; SOUSA, P. H. M.; ARRIAGA, A. M. C.; PRADO, G. M.; MAGALHÃES, C. E. C.; MAIA, G. A.; LEMOS, T. L. G. Bioactive compounds and antioxidant activity of fresh exotic fruits from northeastern Brazil. Food Research International, Essex, v. 44, n. 7, p. 2.155-2.159, 2011.

AOAC - Association of Official Analytical Chemists. Official methods of analysis. $17^{\text {th }}$ ed. Arlington, 2002. $1115 \mathrm{p}$.

BLIGH, E. G.; DYER, W. J. A rapid method of total lipid extraction and purification. Canadian Journal of Biochemistry and Physiology, Toronto, v. 37, n. 8, p. 911-917, 1959.

CARVALHO, F. M. V.; MARCO JÚNIOR, P.; FERREIRA, L. G. The Cerrado into pieces: habitat fragmentation as a function of landscape use in the savannas of central Brazil. Biological Conservation, Essex, v. 142, n. 7, p. 1392-1403, 2009.

CONTRERAS-CALDERÓN, J.; CALDERÓNJAIMES, L.; GUERRA-HERNÁNDEZ, E.; GARCÍAVILLANOVA, B. Antioxidant capacity, phenolic content and vitamin $\mathrm{C}$ in pulp, peel and seed from 24 exotic fruits from Colombia. Food Research International, Essex, v. 44, n. 7, p. 2047-2053, 2011.
IGNAT, I.; VOLF, I.; POPA, V. I. A critical review of methods for characterization of polyphenolic compounds in fruits and vegetables. Food Chemistry, Barking, v. 126, n. 4, p. 1821-1835, 2011.

IOM - Institute of Medicine. Dietary reference intakes research synthesis: workshop summary. Washington: The National Academies Press, 2006. 298 p.

JOSHIPURA, K. J.; HUNG, H. C.; LI, T. Y.; HU, F. B.; RIMM, E. B.; STAMPFER, M. J.; COLDITZ, G.; WILLETT, W. C. Intakes of fruits, vegetables and carbohydrate and the risk of CVD. Public Health Nutrition, Wallingford, v. 12, n. 1, p. 115-121, 2009.

KACZMARCZYK, M. M.; MILLER, M. J.; FREUNDA, G. G. The health benefits of dietary fiber: beyond the usual suspects of type 2 diabetes mellitus, cardiovascular disease and colon cancer. Metabolism, Clinical and Experimental, Baltimore, v. 61, n. 8, p. 1.058-1.066, 2012.

MARQUES, A.; CHICAYBAM, G.; ARAÚJO, M. T.; MANHÃES, L. R. T.; SABAA-SRUR, A. U. O. Composição centesimal e de minerais de casca e polpa de manga (Mangifera indica L.) cv. Tommy Atkins/ Mango rind and pulp (Mangifera indica L.) cv. Tommy Atkins centesimal composition and minerals contents. Revista Brasileira de Fruticultura, Jaboticabal, v. 32, n. 4, p. 1206-1210, 2010.

MARTINS, S.; MUSSATTO, S. I.; MARTÍNEZAVILA, G.; MONTANEZ-SAENZ, J.; AGUILAR, C. N.; TEXEIRA, J. A. Bioactive phenolic compounds: production and extraction by solid-state fermentation. A review. Biotechnology Advances, New York, v. 29, n. 3, p. 365-373, 2011.

OLIVEIRA, M. C.; SANTANA, D. G.; SANTOS, C. M. Biometria de frutos e sementes e emergência de plântulas de duas espécies frutíferas do gênero Campomanesia/Biometrics of fruits and seeds and seedling emergence of two species of fruit of the Campomanesia genus. Revista Brasileira de Fruticultura, Jaboticabal, v. 33, n. 2, p. 446-455, 2011.

PEREIRA, M. C.; STEFFENS, R. S.; JABLONSKI, A.; HERTZ, P. F.; RIOS, A. O.; VIZZOTTO, M.; FLÔRES, S. H. Characterization and antioxidant potential of Brazilian fruits from the Myrtaceae family. Journal of Agricultural and Food Chemistry, Washington, v. 60, n. 12, p. 3061-3067, 2012. 
RE, R.; PELLEGRINI, N.; PROTEGGENTE, A.; PANNALA, A.; YANG, M.; RICE-EVANS, C. Antioxidant activity applying an improved ABTS radical cation decolorization assay. Free Radical Biology \& Medicine, New York, v. 26, n. 9-10, p. 1.231-1.237, 1999 .

ROCHA, W. S.; LOPES, R. M.; SILVA, D. B.; VIEIRA, R. F.; SILVA, J. P.; AGOSTINI-COSTA, T. $\mathrm{S}$. Compostos fenólicos totais e taninos condensados em frutas nativas do cerrado/ Total phenolics and condensed tannins in native fruits from Brazilian savanna. Revista Brasileira de Fruticultura, Jaboticabal, v. 33, n. 4, p. 1215-1221, 2011.

RUFINO, M. S. M.; ALVES, R. E.; BRITO, E. S.; PÉREZ-JIMÉNEZ, J.; SAURA-CALIXTO, F.; MANCINI-FILHO, J. Bioactive compounds and antioxidant capacities of 18 non-traditional tropical fruits from Brazil. Food Chemistry, Barking, v. 121, n. 4, p. 996-1002, 2010.

SHEFTEL, A. D.; MASON, A. B.; PONKA, P. The long history of iron in the universe and in health and disease. Biochimica et Biophysica Acta (BBA) General Subjects, Amsterdam, v. 1820, n. 3, p. 161$187,2012$.

SILVA, M. R.; LACERDA, D. B. C. L.; SANTOS, G. G.; MARTINS, D. M. O. Caracterização química de frutos nativos do cerrado/Chemical characterization of native species of fruits from savana ecosystem. Ciência Rural, Santa Maria, v. 38, n. 6, p. 1790-1793, 2008.
SINGLETON, V. L.; ROSSI, J. A. Colorimetry of total phenolics with phosphomolybidic-phosphotungstic acid reagent. American Journal of Enology and Viticulture, Davis, v. 16, n. 3, p. 144-158, 1965.

SOERJOMATARAM, I.; OOMEN, D.; LEMMENS, V.; OENEMA, A.; BENETOU, V.; TRICHOPOULOU, A.; COEBERGH, J. W.; BARENDREGT, J.; VRIES, E. Increased consumption of fruit and vegetables and future cancer incidence in selected European countries. European Journal of Cancer, Oxford, v. 46, n. 14, p. 2563-2580, 2010.

SOUZA, V. R.; PEREIRA, P. A. P.; QUEIROZ, F.; BORGES, S. V.; CARNEIRO, J. D. S. Determination of bioactive compounds, antioxidant activity and chemical composition of Cerrado Brazilian fruits. Food Chemistry, Barking, v. 134, n. 1, p. 381-386, 2012.

VALLILO,M.I.;LAMARDO,L.C.A.; GARBELOTTI, M. L.; OLIVEIRA, E.; MORENO, P. R. H. Composição química dos frutos de Campomanesia adamantium (Cambessédes) O. BERG/Chemical composition of Campomanesia adamantium (Cambessédes) O. BERG fruits. Ciência e Tecnologia de Alimentos, Campinas, v. 26, n. 4, p. 805-810, 2006. 